\title{
Analysis of statistical data on drop collisions in an aerosol flow during experiments
}

\author{
Dmitry Antonov*, Olga Vysokomornaya, Maxim Piskunov, and Nikita Shlegel \\ National Research Tomsk Polytechnic University, 634050 Tomsk, Russia
}

\begin{abstract}
The study presents a software code developed by using Mathematica. This code allows the automatic analysis of breakup consequences of heterogeneous drops. Moreover, we show how statistical data on drop collisions in a sprayed aerosol flow can be collected using this code. For this purpose, we have compared results of manual handling of video with automatic one.
\end{abstract}

\section{Introduction}

In recent years, due to the steadily rising demand for the potable and fresh industrial water, researchers all over the world try to develop the new methods for enhancing the efficiency of water purification technologies (including the thermal methods) and to improve the current ones. Often, to purify wastewater, engineers use the contact heat exchangers [1,2]. Such technologies are based on evaporation of the contaminated water at high temperatures, usually at about $1000{ }^{\circ} \mathrm{C}$. However, the current models of heat transfer and phase transformations in the system of liquid drop-gaseous medium (or liquid drop heated wall) do not enable the prediction of characteristics for the mentioned processes fully, especially if to consider an effect of solid and liquid impurities. This is because regimes and conditions of heating and evaporation of drops with impurities of different concentrations and types considerably differ from the same characteristics for singlecomponent drops. One of the advanced ways to enhance the phase transformation efficiency is an increase in evaporation surface area by a fine atomization of liquids as well as an explosive breakup of drops. Basically, the explosive breakup is a secondary atomization of liquids directly within the heat-exchange region [3].

The purpose of the study is to demonstrate how the software code developed by the authors is applied to analyze the breakup consequences of heterogeneous drops and to collect statistical data on drop collisions in a sprayed aerosol flow.

\section{Installation and usage}

\subsection{Scenarios of drops collision consequences in an aerosol flow}

\footnotetext{
*Corresponding author: 1aadsdfn@gmail.com
} 
Following the usual terminology, among the most commonly occurring consequences of drops collision can be distinguished such ones as (1) rebound, i.e. drops come close and then fly away from each other (without a direct contact) due to a vapor layer near the surface of drops; most often it is observed in the case of drops with similar sizes at low velocities; (2) coagulation is a merging of drops and further motion in a flow as a one fragment of a liquid; this after-effect often occurs in the case of different sizes of drops at low velocities; (3) split is a collision of two drops with a short-term coalescence and further breakup into two drops in size similar to initial ones; this after-effect is observed in the case of collision of drops with similar momentum; (4) fragmentation is a breakup of the colliding drops into a large number of fragments much smaller as compared to the parent drops; this after-effect generally occurs at high velocities in the case when the colliding drops have far different sizes.

The experimental results allow the conclusion that the thresholds (e.g., the Weber number thresholds) of a certain consequence of drops collisions are conditional. Each of the possible scenarios can occur in any conditions of interacting. However, probabilities of their occurrence can be from less than $1 \%$ to several dozens of percent.

\subsection{Analysis of breakup consequences of heterogeneous drops}

The software code for analyzing the breakup consequences of the heterogeneous drops consists of two main parts. The first part is a search of a binarization threshold. The second one is the main evaluator that finds the fragments of a liquid and determines their sizes in pixels. Each part takes into account a subtraction of background and an elimination of the fragments touching the borders of an image. By using the primary functions of the main evaluator, such as Morphological Binarize and Median filter, the dark objects (childdroplets) are outlined on the light background. Each outlined object represents a spherical droplet with a size of the outlined object. Further, total amount of droplets and size of the outlined child-droplets are determined.

\subsubsection{Binarization threshold search}

A software code for the first stage of video processing is presented below. This stage involves a loading of videos imaging heterogeneous drops breakup and background (Fig. 1).

In[1]:= file = SystemDialogInput["FileOpen", WindowTitle -> "Select VIDEO"];

dat=Import@ file;

dir = SetDirectory[DirectoryName@file];

bkg=Import@SystemDialogInput["FileOpen", dir,

WindowTitle -> "Select BACKGROUND"];

Next, a deletion of the small fragments as well as the fragments on the image borders is accomplished. In addition, using the Median Method a morphologic binarization is performed.

In[2]:= fig = ImageTake[ImageDifference[ImageAdjust@dat[[20]],

bkg[[5]]], $\{1,-1\},\{1,-1\}]$;

so=Framed@Show[\#, ImageSize -> 700] \&;

Manipulate[Row[so /@ ffig, DeleteBorderComponents@

DeleteSmallComponents[ColorReplace[MorphologicalBinarize

[fig, i, Method -> "Median"], 
$\{$ White -> Black, Black -> White $\}], 2]\}],\{$ i, $0,0.8,0.01\}]$

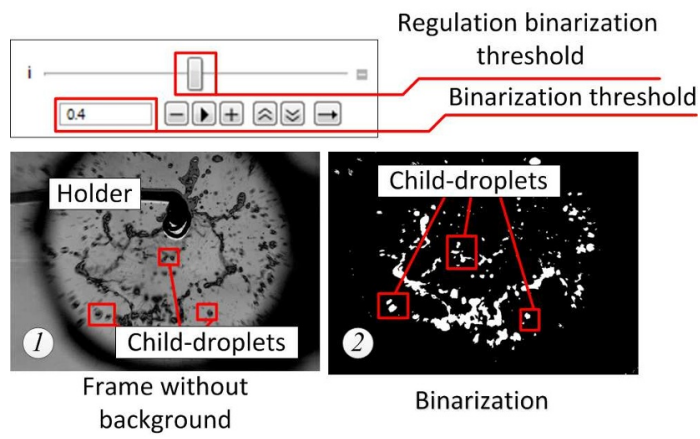

Fig. 1. The first stage of processing a video recorded during experiments: the binarization threshold search.

\subsubsection{Main evaluator}

The main software code for defining the breakup consequences of the heterogeneous drops is shown below.

$\operatorname{In}[1]:=$ binpar $=0.4$

LaunchKernels[];

data=ImageTake[ImageDifference[ImageAdjust@\#, bkg],

$\{1,-1\},\{1,-1\}] \& / @$ Import[file];

PrintTemporary[Style["Frame-data ", Red], " <= Imported"];

DistributeDefinitions[data];

ddt2 = ParallelTable[DeleteBorderComponents@ DeleteSmallComponents

[ColorReplace[MorphologicalBinarize[data[[k]], binpar, Method -> "Median"],

\{White -> Black, Black -> White \}], 2], \{k,1, Length@data\}];

ClearAll[data]; Share[];

CloseKernels[]; LaunchKernels[];

PrintTemporary[Style["- Background removed ", Blue]];

obj=ComponentMeasurements[\#,\{(*"Centroid",*)"Length" $\}][[$ All,2]]\&/@ ddt2;

PrintTemporary[Style["- Object positions and sizes detected", Blue]];

CloseKernels[].

\subsection{A collecting statistical data on drops collisions in a sprayed aerosol flow}

The first stage of processing images, i.e. the binarization threshold search, to collect statistical data on drops collisions in a sprayed aerosol flow is identical with the processing procedure described in subsection 2.1.1. The second stage includes not only estimating the number of droplets on an image but also establishing a fact of drops collisions. A software implementation of drops collisions determination procedure is based on creating the Distance Matrix. When analyzing videos, the initial data include a scale factor $(\mathrm{mm} / \mathrm{px})$, a binarization threshold, and a proximity threshold.

\section{Results and discussions}

Fig. 2a presents a diagram of child-droplets distribution after breakup of a heterogeneous drop during the convective heating at $T_{\text {air }}=350{ }^{\circ} \mathrm{C}$ and $U_{\text {air }}=2 \mathrm{~m} / \mathrm{s}$. Data have been obtained 
by using Mathematica. The size is estimated in px. Fig. 2b shows a comparison of ratios of the evaporation surface areas before and after breakup under manual and automatic handling. The results adequately correlate. The distinction is less than $6 \%$. This distinction is mainly due to the software limitation concerning the drops detection out of focus.

Fig. 3 demonstrates how the software code can promote to collect the statistical data on collisions in the sprayed flow. The software code finds the couples of drops, which are at a distance less than the proximity threshold, outlines them in the form of circles, and estimates a distance between centers of their mass. When you point to any of the outlined couples of drops, the software demonstrates the results about coordinates, velocities, and sizes, as well as shows an angle between motion directions of drops in the sprayed flow.
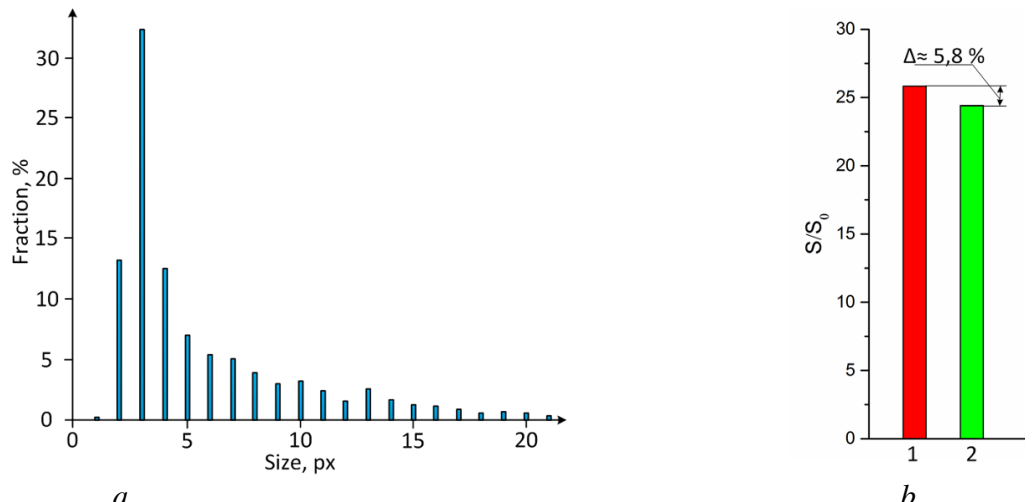

Fig. 2. Analysis of a breakup of the heterogeneous drop in the Mathematica software: $a$ - diagram of the size distribution of child-droplets; $b$ - comparison of ratios of the evaporation surface areas before and after breakup under manual (1) and automatic (2) handling of video

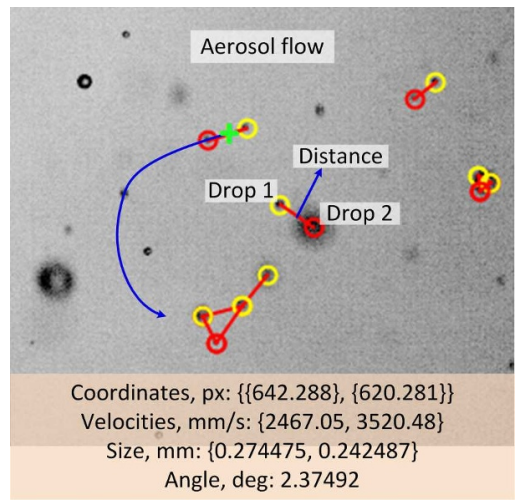

Fig.3. Example of processing the image with the collisions of drops, the proximity threshold is $30 \mathrm{px}$.

\section{Conclusion}

An instrument for numerical simulation of features and mechanisms of interacting between water droplets in aerosol flows will enable to predict reliably characteristics for mixing, heating and evaporation of a liquid in gaseous media for a wide group of practical applications (thermal water treatment, polydisperse fire extinguishing, water-fuel emulsions, gas-vapor heat carriers, etc.). The developed software code can be used to analyze large volumes of data on collisions and breakup of droplets (including heterogeneous ones) in an aerosol flow. A difference between the results of manual and 
automatic handling is 5-10\%. This fact allows the conclusion about a good efficiency of the developed software code under a significant reduction in time required for processing of experimental results.

This study was financially supported by the Russian Science Foundation (project 18-71-10002).

\section{References}

1. S.A. Kalogirou, Prog. Energy Combust. Sci., 31, 3 (2005), DOI: 10.1016/j.pecs.2005.03.001

2. M.A. Shannon, P.W. Bohn, M. Elimelech, J.G. Georgiadis, B.J. Mariñas, A.M. Mayes, Nature, 452 (2008), DOI: 10.1038/nature06599

3. M.V. Piskunov, P.A. Strizhak, Exp. Therm. Fluid Sci., 91 (2018), DOI: 10.1016/j.expthermflusci.2017.10.018 